\title{
Inguinal Lymphadenectomy
}

National Cancer Institute

\section{Source}

National Cancer Institute. Inguinal Lymphadenectomy. NCI Thesaurus. Code C51619.

Surgical removal of one or more inguinal lymph nodes. 